\title{
Health-related quality of life and distress in cancer patients: results from a large randomised study
}

\author{
B Johansson*, , Y Brandberg ${ }^{2}$, M Hellbom ${ }^{3}$, C Persson', L-M Petersson ${ }^{4}$, G Berglund ${ }^{5}$ and B Glimelius ${ }^{1,2}$ \\ 'Department of Oncology, Radiology and Clinical Immunology, Uppsala University, Uppsala, Sweden; ${ }^{2}$ Department of Oncology-Pathology, Karolinska \\ Institutet, Stockholm, Sweden; ${ }^{3}$ Department of Oncology, Lund University Hospital, Lund, Sweden; ${ }^{4}$ Department of Clinical Neuroscience, Section for \\ Personal Injury Prevention, Karolinska Institutet, Stockholm, Sweden; ${ }^{5}$ Department of Public Health and Caring Sciences, Uppsala University, Uppsala, \\ Sweden
}

To compare the effectiveness of individual support, group rehabilitation and a combination of the two in improving health-related quality of life $(\mathrm{HRQOL})$ and psychological well-being in cancer patients during 24 months after diagnosis, as compared with standard care (SC). Furthermore, to compare the study sample and a random sample of the Swedish population with regard to HRQOL. A total of $48 \mathrm{I}$ consecutive patients, newly diagnosed with cancer, were randomly assigned to one of the four alternatives. Data on HRQOL and psychological well-being were collected at baseline and after 3, 6, 12 and 24 months. The interventions did not improve $\mathrm{HRQOL}$ or psychological well-being, as compared with SC. At 3 months, the study sample reported an HRQOL comparable with the normal population. Many cancer patients are able to manage their cancer-related concerns with the support available from SC. However, it is reasonable to assume that the findings suffer from a lack of data from especially vulnerable patients and a possible Hawthorne effect. It cannot be concluded that cancer patients have no need for additional psychosocial interventions. Future projects should include screening and target interventions for those at risk for significant and prolonged psychological distress.

British Journal of Cancer (2008) 99, 1975-1983. doi:I0.1038/sj.bjc.6604789 www.bjcancer.com

Published online 18 November 2008

(c) 2008 Cancer Research UK

Keywords: health-related quality of life; distress; psychosocial support; group rehabilitation

Cancer and its treatment influence health-related quality of life (HRQOL) in several domains, and the assessment of HRQOL is crucial to efforts to improve clinical outcomes (Arndt et al, 2004; Stanton et al, 2007). Many cancer patients experience psychological distress (Nordin and Glimelius, 1997; Burgess et al, 2005; Korfage et al, 2006; Strong et al, 2007), and a relatively high incidence of post-traumatic stress disorder symptoms has been observed (Kangas et al, 2005). A large number of studies have evaluated the effects of psychosocial interventions on psychological distress and HRQOL (van't Spijker et al, 1997; Newell et al, 2002). However, it is still unclear whether individual or group support is more effective in relieving problems of anxiety and depression (Sheard and Maguire, 1999; Osborn et al, 2006). Different types of interventions have been evaluated in different diagnostic groups and at different times in the illness trajectory (Arving et al, 2007; May et al, 2008), which makes it difficult to assess the clinical value of the trial results. In addition, a majority of the studies include small samples, which jeopardises the

\footnotetext{
*Correspondence: Dr B Johansson, Department of Oncology, Radiology and Clinical Immunology, Uppsala University, Uppsala SE-75I 85, Sweden; E-mail: birgitta.johansson@onkologi.uu.se

Presentation list: Seventh World Congress of Psycho-Oncology, Copenhagen, 2004

Received 21 July 2008; revised 15 October 2008; accepted 24 October 2008; published online 18 November 2008
}

possibilities of detecting small but clinically relevant differences between groups.

Techniques from cognitive-behavioural therapy may be effective in relieving psychological distress and treatment side effects in cancer patients (Uitterhoeve et al, 2004; Osborn et al, 2006). Methods such as distractions, relaxation and cognitive restructuring have been used to reduce, for example anxiety, depression, nausea/vomiting and pain.

Involuntary weight loss is another well-recognised problem affecting HRQOL in cancer patients (Persson et al, 2002). This problem may be decreased through early nutritional assessment and subsequent interventions for risk patients (Ravasco et al, 2005). Another important aspect of cancer care is the co-ordination between different levels of the care system, to improve continuity and reduce the patients' need for emergency and hospital care (Burge et al, 2003).

The Support-Care-Rehabilitation (SCR) project was carried out between 1993 and 1997. The overall aim was to investigate whether individual support begun at diagnosis or group support during the rehabilitation period, or a combination of both, could help relieve short- and long-term physical and psychosocial problems. Patients were randomised among four groups in a $2 \times 2$ design: (1) individual support (IS) starting immediately, (2) group rehabilitation (GR), starting approximately 3 months after diagnosis, (3) a combination of IS and GR (ISGR) and (4) standard care (SC). The follow-up period was 24 months for all patients.

The aim of this study was to evaluate the effects of IS, GR and ISGR on HRQOL and psychological well-being in cancer patients at 
3, 6, 12 and 24 months after diagnosis, as compared with SC. A further aim was to compare the study sample and a random sample of the Swedish population with regard to HRQOL.

\section{MATERIALS AND METHODS}

\section{Patients}

Consecutive patients with newly diagnosed ( $<3$ months) prostate, gastrointestinal $(\mathrm{GI}=$ colorectal or gastric) or breast cancer were included. In addition, women with a mammography finding requiring surgery could also be included. Exclusion criteria were the Karnofsky performance status $<40$, an earlier cancer diagnosis, inability to communicate in Swedish and participation in an ongoing randomised trial for patients with localised prostate cancer $(n=59)$. Of 756 eligible patients, $73(10 \%)$ were not approached due to uncertainty about the diagnosis or adminis- trative failure (Figure 1). A total of 202 patients (26\%) rejected participation. The most common reasons for this were 'no interest' $(n=80)$ and 'too far to travel' $(n=51)$. Patients who rejected participation were older (mean: 71 years) than those who accepted participation (mean: 62 years, $P<0.001$ ). This difference was evident in all diagnostic groups. Of the 527 included patients, 42 were excluded due to a benign breast tumour at surgery. Four patients were excluded due to an erroneous cancer diagnosis $(n=2)$, senility $(n=1)$ or inclusion $\geqslant 3$ months from diagnosis $(n=1)$. Of all eligible patients, $223(76 \%)$ with breast cancer, 140 (64\%) with GI cancer (colorectal, $n=105$ and gastric cancer, $n=37)$ and 118 patients (49\%) with prostate cancer were included. Thus, of total 756 eligible patients, 481 (64\%) agreed to participate, using a procedure approved by the local Research Ethics Committee (Figure 1).

A total of $142(30 \%)$ patients did not complete the entire 24-month follow-up period (Figure 1). Seventy-two (51\%) of those died and $70(49 \%)$ rejected further participation. Those who

\begin{tabular}{|c|c|c|c|}
\hline & \multicolumn{2}{|c|}{$\begin{array}{l}\text { Eligible patients, } n=760 \\
\text { Not approached, } n=73 \\
\text { Rejected participation, } n=202\end{array}$} & \\
\hline & \multicolumn{2}{|c|}{$\begin{array}{l}\text { Agreed to participate, } n=527 \\
\text { Excluded patients, } n=46 \\
\text { Benign breast tumour, } n=42 \\
\text { Other reasons, } n=4\end{array}$} & \\
\hline & \multicolumn{2}{|c|}{ Randomised eligible, $n=481$} & \\
\hline ISGR, $n=117$ & $\begin{array}{l}\text { Individual support } \\
\text { (IS), } n=134\end{array}$ & $\begin{array}{l}\text { Group rehabilitation } \\
(\mathrm{GR}), n=104\end{array}$ & $\begin{array}{l}\text { Standard care (SC), } \\
n=126\end{array}$ \\
\hline \multicolumn{4}{|c|}{ Baseline } \\
\hline $\begin{array}{l}\text { Continued participation, } \\
n=114 \\
\text { Deviated from protocol: } \\
\text { Rejected participation, } n=3 \\
\text { Deceased, } n=0\end{array}$ & \begin{tabular}{|l|} 
Continued participation, \\
$n=128$ \\
Deviated from protocol: \\
Rejected participation, $n=5$ \\
Deceased, $n=1$ \\
\end{tabular} & \begin{tabular}{|l|} 
Continued participation, \\
$n=99$ \\
Deviated from protocol: \\
Rejected participation, $n=5$ \\
Deceased, $n=0$ \\
\end{tabular} & $\begin{array}{l}\text { Continued participation, } \\
n=116 \\
\text { Deviated from protocol: } \\
\text { Rejected participation, } n=7 \\
\text { Deceased, } n=0\end{array}$ \\
\hline \multicolumn{4}{|c|}{3 months } \\
\hline $\begin{array}{l}\text { Continued participation, } \\
n=104 \\
\text { Deviated from protocol: } \\
\text { Rejected participation, } n=11 \\
\text { Deceased, } n=0\end{array}$ & \begin{tabular}{|l|} 
Continued participation, \\
$n=109$ \\
Deviated from protocol: \\
Rejected participation, $n=11$ \\
Deceased, $n=4$ \\
\end{tabular} & \begin{tabular}{|l|} 
Continued participation, \\
$n=88$ \\
Deviated from protocol: \\
Rejected participation, $n=14$ \\
Deceased, $n=1$ \\
\end{tabular} & $\begin{array}{l}\text { Continued participation, } \\
n=103 \\
\text { Deviated from protocol: } \\
\text { Rejected participation, } n=11 \\
\text { Deceased, } n=9 \\
\end{array}$ \\
\hline \multicolumn{4}{|c|}{6 months } \\
\hline $\begin{array}{l}\text { Continued participation, } \\
n=101 \\
\text { Deviated from protocol: } \\
\text { Rejected participation, } n=14 \\
\text { Deceased, } n=1\end{array}$ & \begin{tabular}{|l|} 
Continued participation, \\
$n=105$ \\
Deviated from protocol: \\
Rejected participation, $n=13$ \\
Deceased, $n=14$ \\
\end{tabular} & \begin{tabular}{|l|} 
Continued participation, \\
$n=82$ \\
Deviated from protocol: \\
Rejected participation, $n=16$ \\
Deceased, $n=2$
\end{tabular} & $\begin{array}{l}\text { Continued participation, } \\
n=98 \\
\text { Deviated from protocol: } \\
\text { Rejected participation, } n=16 \\
\text { Deceased, } n=11 \\
\end{array}$ \\
\hline \multicolumn{4}{|c|}{12 months } \\
\hline $\begin{array}{l}\text { Continued participation, } \\
n=95 \\
\text { Deviated from protocol: } \\
\text { Rejected participation, } n=16 \\
\text { Deceased, } n=6\end{array}$ & \begin{tabular}{|l|} 
Continued participation, \\
$n=98$ \\
Deviated from protocol: \\
Rejected participation, $n=16$ \\
Deceased, $n=20$ \\
\end{tabular} & \begin{tabular}{|l|} 
Continued participation, \\
$n=84$ \\
Deviated from protocol: \\
Rejected participation, $n=16$ \\
Deceased, $n=3$ \\
\end{tabular} & $\begin{array}{l}\text { Continued participation, } \\
n=93 \\
\text { Deviated from protocol: } \\
\text { Rejected participation, } n=17 \\
\text { Deceased, } n=16 \\
\end{array}$ \\
\hline \multicolumn{4}{|c|}{24 months } \\
\hline $\begin{array}{l}\text { Included in analyses, } \\
n=89 \\
\text { Deviated from protocol: } \\
\text { Rejected participation, } n=14 \\
\text { Deceased, } n=14\end{array}$ & \begin{tabular}{|l|} 
Included in analyses, \\
$n=91$ \\
Deviated from protocol: \\
Rejected participation, $n=19$ \\
Deceased, $n=24$ \\
\end{tabular} & \begin{tabular}{|l|} 
Included in analyses, \\
$n=80$ \\
Deviated from protocol: \\
Rejected participation, $n=16$ \\
Deceased, $n=8$ \\
\end{tabular} & $\begin{array}{l}\text { Included in analyses, } \\
n=79 \\
\text { Deviated from protocol: } \\
\text { Rejected participation, } n=21 \\
\text { Deceased, } n=26 \\
\end{array}$ \\
\hline
\end{tabular}

Figure I Participant flow. 
deviated from the protocol were older and included a larger proportion with advanced disease (Advanced disease was GI cancer with distant metastases (M1); prostate cancer with T-stage 4 , lymph node and/or distant metastases ( + and/or M1) and breast cancer with locally advanced cancer (T3-T4) and/or $>7$ positive axillary nodes or distant metastases (M1).), as compared with those who remained in the project (mean age 67 years compared with 63 years, $P<0.001$; advanced disease $n=64,45 \%$ compared with $n=46,14 \%, P<0.001)$.

\section{Randomisation}

Randomisation was stratified for diagnosis and stage. Patients were randomised by an independent oncologic centre (computergenerated allocation schedule) to one of the four alternatives. Colorectal and gastric cancer patients who had non-curable disease (M1, short expected survival) were randomised between only IS and SC (no rehabilitation condition). Thus, there was a higher proportion of advanced GI cancer patients in IS and SC, as compared with GR and ISGR (Table 1). With that exception, there were no differences between treatment groups with regard to baseline demographic and medical characteristics.

\section{Interventions}

All interventions have been described in detail elsewhere (Hellbom et al, 1998; Johansson et al, 1999; Petersson et al, 2000; Persson et al, 2002).

IS Individual support began as soon as possible after randomisation and consisted of individual psychological support, intensified primary health care and nutritional support. All patients in IS were contacted by a project psychologist (Hellbom et al, 1998). Current problems identified jointly by the patient and the psychologist were the focus of the intervention. Techniques used were derived from cognitive behaviour therapy, including relaxation techniques, identification and challenging of negative automatic thoughts and activity scheduling and daily planning. If no problems were identified, the contact was terminated, but the patient was permitted to contact the psychologist when problems arise. Most sessions were conducted face to face at the project agency. However, a number of sessions were conducted by telephone because of long travelling distances or disease- or treatmentrelated problems. The median number of psychologist contacts was 3 (minimum-maximum: 1-24). The psychologists in the project received regular supervision throughout the study period.

Table I Baseline demographic and medical characteristics of the study sample, $n=48$

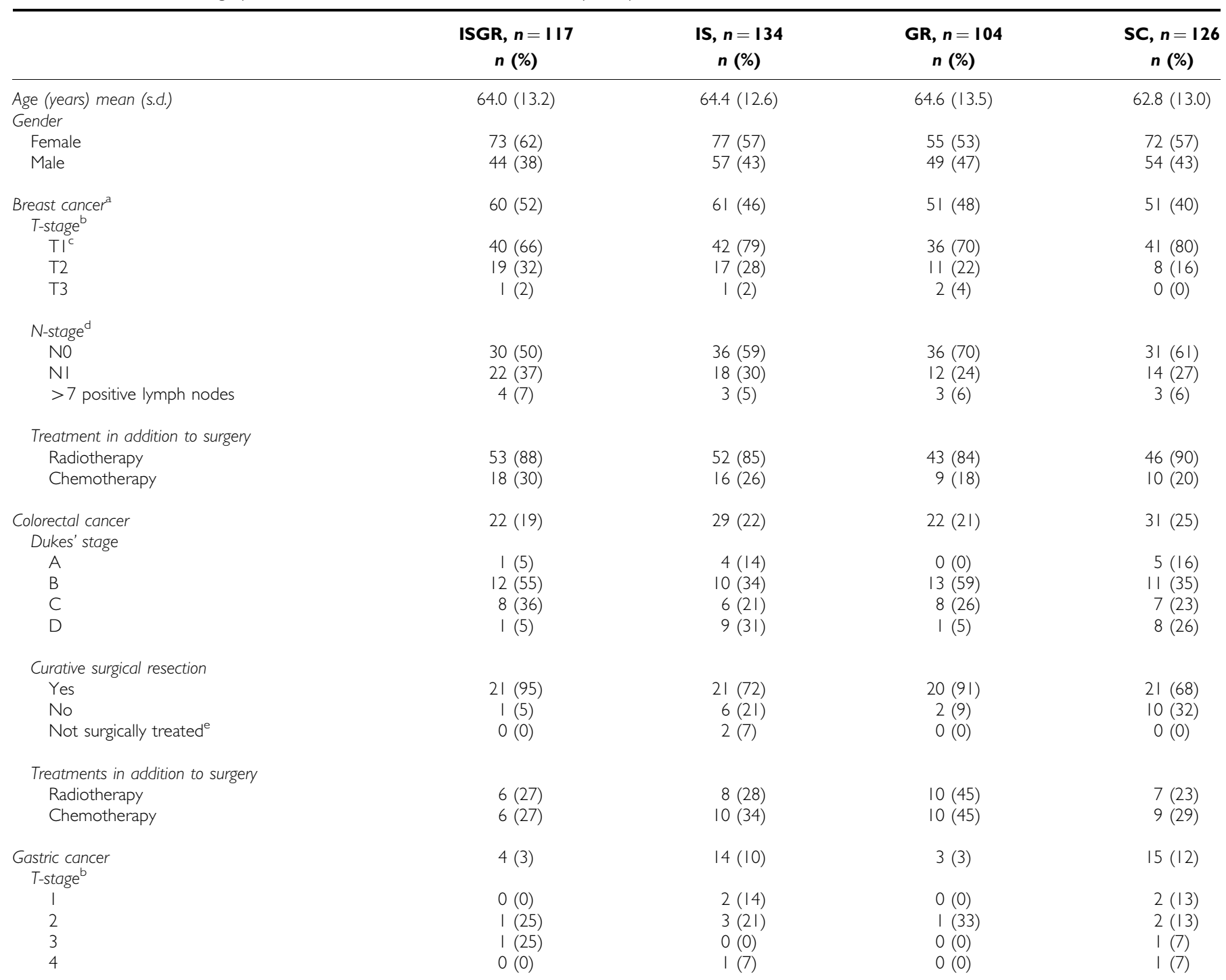


Table I (Continued)

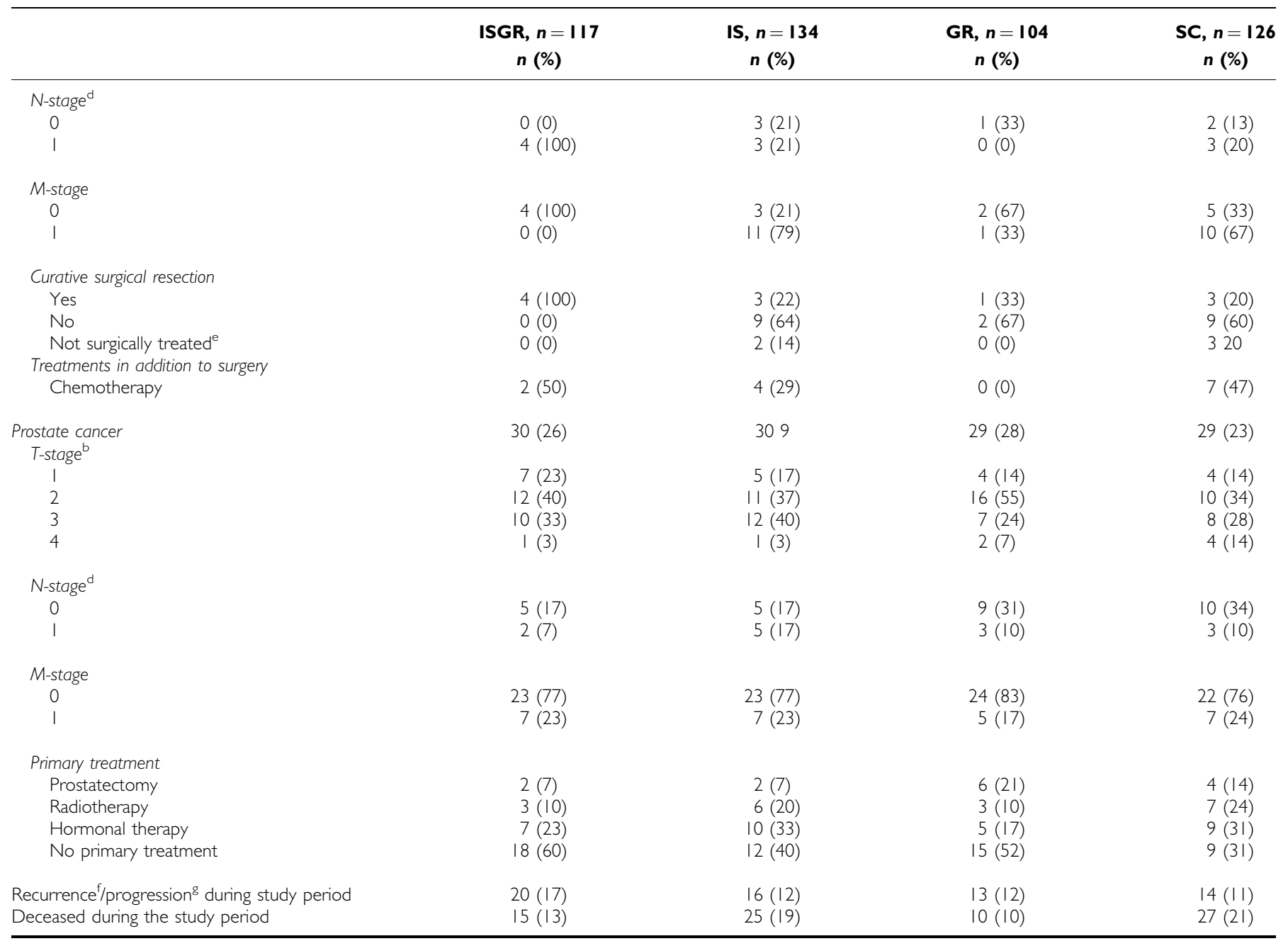

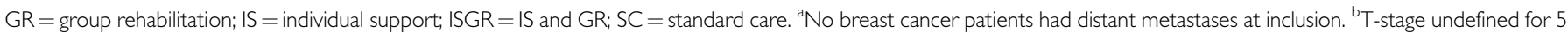
breast cancer, 2 I gastric cancer and 5 prostate cancer patients. Including cancer in situ, $n=17$. d N-stage undefined for 24 breast cancer, 20 gastric cancer and 77 prostate cancer patients. ${ }^{e}$ Patients with distant metastases. ${ }^{f}$ Nine patients were affected by a new cancer. ${ }^{\text {gP }}$ rogression for prostate cancer only.

Intensified primary health care meant that each patient was referred to the home care nurse in their neighbourhood (Johansson et al, 1999, 2001). The patient's general practitioner (GP) was also informed about the cancer diagnosis and the referral to the nurse. GPs and home care nurses received copies of the medical records each time the patient was discharged from hospital or had visited a specialist outpatient clinic. Education in cancer care was arranged during the course of the trial for nurses and GPs. In addition, GPs and home care nurses with patients randomised to IS were offered supervision by a multi-professional oncology team. Overall, $90 \%$ of IS patients reported home care nurse follow-up contacts, as compared with $26 \%$ of non-IS patients.

Patients with GI cancer also received nutritional support (Persson et $a l, 2002)$. A dietician made a dietary assessment including calculation of the dietary intake as soon as possible after diagnosis. After assessment, the dietician gave nutritional advice. When needed, supplements and nutritional enrichment were prescribed. The next assessment was scheduled 2-3 months later. Assessments were conducted by telephone or face to face when the patient had a period of in-patient care or visited the outpatient clinic.

GR Group rehabilitation started approximately 3 months after randomisation and comprised 8 weekly sessions and a booster session after 2 months (Petersson et al, 2000). The groups consisted of 3-9 participants. Group rehabilitation implied that the patient visited the project agency. A psychologist, physiotherapist and oncology nurse conducted the meetings. Sessions included cognitive behavioural techniques, light physical training and relaxation. In two of the sessions, a physician presented information about cancer and cancer treatment, and a dietician provided dietary advice. All sessions offered opportunities to disclose and discuss concerns with group leaders and members. Overall, $80 \%$ of the patients participated in five or more sessions.

IS and GR Individual support and GR meant that the patients received IS followed by GR. Individual psychological support was (with few exceptions) terminated before GR began. A total of 132 $(67 \%)$ of 196 invited patients participated in GR. A significantly larger proportion of patients randomised to ISGR (42 out of 106) declined GR participation as compared with patients randomised to GR only ( 22 out of 90$)\left(\chi^{2}=5.10\right.$; d.f. $\left.=1 ; P<0.05\right)$.

Standard care Standard care did not include regular follow-ups by a dietician or medical social worker. However, the patients could be referred to such services if the physician or the nurse judged it necessary or if the patient made a specific request. 
Psychologists were not available at the surgery or oncology departments. Rehabilitation programmes or support groups were not arranged. Referrals to home care nurses or GPs were rare, as they usually are in routine care (Johansson et al, 1999).

\section{Data collection}

Background data Data on patients' age, diagnosis, stage of disease and treatment were collected from the medical records.

Points of assessments The patients completed the baseline assessment before being informed about the randomisation result. Subsequent assessments took place 3, 6, 12 and 24 months after inclusion. The research nurse who informed the patients about the project gave them the baseline questionnaires together with a prepaid envelope. At subsequent assessments, the patients were contacted by one of the investigators by phone. The investigator gave instructions and then mailed the questionnaires, written instructions and a prepaid envelope to the patient. Returned questionnaires were checked for incomplete responses, and in such cases the investigator contacted the patients by phone to complete the questionnaire.

Questionnaires This study includes data from the well-known questionnaires European Organization for Research and Treatment of Cancer-Quality of Life Questionnaire (EORTC QLQ-C30) (version 1) (Aaronson et al, 1993; Fayers et al, 2001), the Hospital Anxiety and Depression Scale (HADS) (Zigmond and Snaith, 1983) and the Impact of Event Scale (IES) (Horowitz et al, 1979). All three were included at all points of assessment.

HRQOL in the Swedish population Normative data on EORTC QLQ-C30 (Michelson et al, 2000) were used for the comparison of HRQOL in the study sample and the Swedish population. The EORTC QLQ-C30 was sent to 3919 adults and completed by 3069 (78\%), 1619 (53\%) women and $1450(47 \%)$ men. The mean age for both genders was 51.2 years.

Statistical analyses The statistical analyses were performed with the SPSS v. 12.0.1. Substitution of missing values was made with the mean of each patient's responses, provided that at least half of the subscale items had been completed (Fayers et al, 2001). A one-way ANOVA with repeated measures was used to analyse the effects of IS on HRQOL, anxiety, depression and post-traumatic distress at 3 months. A two-way factorial ANOVA (IS/non-IS, and GR/non-GR) with repeated measures was used to analyse treatment-by-time interactions at 6,12 and 24 months, using 3-month data as a second baseline. Patients with non-curable GI cancer, who were not randomised to GR, were excluded from the latter analyses. Pairwise comparisons, in case of statistically significant main or interactions effects, were performed with a Bonferroni correction. Additional analyses including age as a covariate in all models were also conducted. However, they did not yield differing results (data not presented). Power calculations were undertaken using a power of $80 \%$ with a 0.05 two-sided significance level. A sample size of 75 in each group was required to detect a mean difference of 7.5 (s.d.=23.0) in QLQ-C30 subscales. The corresponding values for the HADS anxiety or depression were a sample size of 57 and a mean difference of 1.5 $($ s.d. $=4.0)$, and for the IES 72 in each group and mean difference of 3 (s.d. =9.0).

Differences in scores for the EORTC QLQ questionnaires from baseline to the 24-month assessment were interpreted in terms of clinical relevance, according to Osoba et al (1998), as small $(5-10 p)$, moderate $(11-19 p)$ and large $(>20 p)$ changes. Improvements in levels of depression, anxiety and distress were interpreted as the number with a lower level of problem according to recommended cutoff scores for the HADS and the IES, respectively. In addition, one-way ANOVA was performed to assess initial differences between groups at baseline and 3 months, and $t$-tests for the assessment of differences between participants and dropouts.

Comparisons with normative HRQOL data were adjusted for gender and age (Hjermstad et al, 1998). A one-sample $t$-test was used to compare means between the study sample and the normal population.

\section{RESULTS}

Patients who did not complete all five assessments $(n=142)$ had a worse HRQOL at baseline compared with those who did. There were differences with regard to the EORTC QLQ-C30 subscales global health status, physical functioning, role functioning, social functioning, fatigue, nausea and vomiting, pain, dyspnoea, appetite loss, constipation and diarrhoea (mean differences $=3-13$, $P<0.001$ to $<0.05$ ), and with regard to the HAD Depression subscale (means $=5.1$ and $3.5, P<0.001$ ).

Data on the EORTC QLQ-C30 at all points of assessments are presented in Table 2 and data on the HADS and IES in Table 3. There were no baseline differences between IS and SC. Group rehabilitation patients had lower levels of constipation (mean $=5.9$, s.d. $=16.4)$ at 3 months as compared with non-GR patients $($ mean $=10.7$, s.d. $=23.5)(\mathrm{F}=5.4$, d.f. $=1, P=0.02)$.

\section{Interaction effects}

Role functioning improved from 3 to 6 and 12 months (mean $=80.6,82.2$, 90.6; s.d. $=28.0,29.3,21.9)$ and deteriorated slightly at 24 months $($ mean $=85.6$, s.d. $=28.3$ ) in GR patients, whereas it improved at all points of assessment (mean $=85.4-88.1$, s.d. $=25.4-25.3)$ in non-GR patients $(\mathrm{F}=2.7$, d.f. $=3 / 954$, $P=0.045)$. However, there were no statistically significant differences in the pairwise comparisons. Appetite loss decreased from 3 to 6 months $($ mean $=5.1-3.7$, s.d. $=14.2-11.1)$, worsened at 12 months $($ mean $=5.8$, s.d. $=16.0)$ and decreased again at 24 months $($ mean $=4.1$, s.d. $=12.7)$ in GR patients, whereas it improved from 3 to 6 and 12 months (mean=8.1, 4.0, 2.4; s.d. $=17.7,12.6,10.8)$ and worsened at 24 months $($ mean $=2.8$, s.d. $=10.7)$ in non-GR patients $(\mathrm{F}=4.1$, d.f. $=3 / 963, P=0.007)$. Pairwise comparisons revealed a statistically significant mean difference between GR and non-GR patients at 12 months $($ mean $=5.8$ vs 2.4$)$. There were no other treatment-by-time interactions and no interaction effects of IS and GR.

\section{Main effects of treatments}

Individual support patients had a higher level of cognitive functioning $($ mean $=87.4$, s.d. $=14.2)$ as compared with non-IS patients $($ mean $=84.0$, s.d. $=16.4)(\mathrm{F}=4.6$, d.f. $=1 / 321, P=0.046)$ from 3 to 24 months. There were no other main effects of treatments.

\section{Effect of time}

The levels of problems were generally low with the exception of a slightly deteriorated HRQOL at baseline, as compared with the following points of assessment (Tables 2 and 3). Global quality of life, emotional functioning, cognitive functioning, pain, insomnia, appetite loss, anxiety, depression, intrusion and avoidance improved from baseline to 3 months. Financial difficulties worsened from baseline to 3 months. Global quality of life, physical functioning, role functioning, emotional functioning, social functioning, fatigue, nausea/vomiting, appetite loss, diarrhoea, financial difficulties, and depression improved from 3 to 24 months. Intrusion and avoidance improved from baseline to 3 and 
Table 2 Mean values (s.d.) for EORTC QLQ C-30 subscales for all groups (IS, $n=242-9$ |; ISGR, $n=1 \mid$ 4 -89; GR, $n=99-80 ;$ SC, $n=2$ I5-79) at five points of assessment

\begin{tabular}{|c|c|c|c|c|c|c|c|c|c|c|}
\hline Subscales & Treatment & Baseline & 3 months & Treatment & 3 months & 6 months & 12 months & 24 months & Difference & F $(P \text {-value })^{a}$ \\
\hline \multirow[t]{4}{*}{ Global health status $(\mathrm{q} l)^{\mathrm{b}, \mathrm{c}}$} & IS & $65(23)$ & $70(22)$ & IS & $69(23)$ & $70(20)$ & $74(20)$ & $74(17)$ & $\mathrm{S}$ & \\
\hline & & & & ISGR & $72(21)$ & $74(20)$ & $74(21)$ & $75(19)$ & $S$ & 1.0 \\
\hline & SC & $63(23)$ & $70(20)$ & GR & 71 (19) & $72(19)$ & 73 (19) & 75 (19) & $M$ & $(0.4)$ \\
\hline & & & & SC & $69(21)$ & $71(21)$ & $70(23)$ & $74(22)$ & M & \\
\hline \multirow[t]{4}{*}{ Physical functioning $(\mathrm{Pf})^{c}$} & IS & 83 (22) & $83(21)$ & IS & $84(19)$ & $84(20)$ & 84 (22) & $85(21)$ & & \\
\hline & & & & ISGR & $82(22)$ & $86(19)$ & $86(20)$ & $86(20)$ & & 0.1 \\
\hline & SC & $81(25)$ & $83(20)$ & GR & $82(19)$ & $87(15)$ & $88(17)$ & $87(20)$ & & $(0.9)$ \\
\hline & & & & SC & $84(21)$ & $83(21)$ & $86(19)$ & $85(22)$ & & \\
\hline \multirow[t]{4}{*}{ Role functioning $(\mathrm{rf})^{\mathrm{c}}$} & IS & $80(3 \mid)$ & $80(30)$ & IS & $82(30)$ & $83(28)$ & 87 (25) & $86(28)$ & S & \\
\hline & & & & $I S G R^{d}$ & $78(29)$ & $82(30)$ & $87(24)$ & $84(29)$ & $\mathrm{S}$ & 0.5 \\
\hline & SC & 78 (34) & $82(28)$ & $G^{d}$ & $83(26)$ & $82(29)$ & $92(23)$ & $88(27)$ & $M$ & $(0.7)$ \\
\hline & & & & SC & 81 (29) & $85(28)$ & $87(25)$ & $90(23)$ & M & \\
\hline \multirow[t]{4}{*}{ Emotional functioning $(\mathrm{ef})^{\mathrm{b}, \mathrm{c}}$} & IS & $74(23)$ & $84(18)$ & IS & $83(18)$ & $83(18)$ & $85(17)$ & $87(17)$ & M & \\
\hline & & & & ISGR & $84(19)$ & $86(18)$ & $87(18)$ & $87(18)$ & M & 0.3 \\
\hline & SC & 75 (23) & $82(19)$ & GR & $83(20)$ & $85(18)$ & $85(17)$ & $86(18)$ & $M$ & $(0.8)$ \\
\hline & & & & SC & $81(19)$ & $85(19)$ & $85(17)$ & $86(20)$ & $M$ & \\
\hline \multirow[t]{4}{*}{ Cognitive functioning $(c f)^{b}$} & IS & $84(20)$ & $86(18)$ & $\mathrm{IS}^{\mathrm{e}}$ & $87(19)$ & $86(19)$ & $86(15)$ & $88(16)$ & & \\
\hline & & & & $I^{e} G R$ & $86(16)$ & $87(15)$ & $87(18)$ & $88(17)$ & & 0.3 \\
\hline & SC & $82(21)$ & $83(19)$ & GR & $84(18)$ & $84(20)$ & $86(18)$ & $86(19)$ & & $(0.8)$ \\
\hline & & & & SC & $83(19)$ & $82(19)$ & $84(19)$ & $82(20)$ & & \\
\hline \multirow[t]{4}{*}{ Social functioning $(s f)^{c}$} & IS & $84(24)$ & $88(20)$ & IS & $87(20)$ & $90(17)$ & $92(15)$ & $91(18)$ & s & \\
\hline & & & & ISGR & $88(20)$ & $92(16)$ & $93(16)$ & $93(15)$ & S & 0.9 \\
\hline & SC & $82(25)$ & $84(23)$ & GR & $86(21)$ & $89(20)$ & $91(17)$ & $89(20)$ & S & $(0.4)$ \\
\hline & & & & SC & $82(24)$ & $86(22)$ & $91(18)$ & $90(20)$ & $\mathrm{S}$ & \\
\hline \multirow[t]{4}{*}{ Fatigue $(\mathrm{fa})^{c}$} & IS & $33(27)$ & $30(24)$ & IS & $29(24)$ & $27(24)$ & $21(22)$ & $21(22)$ & $M$ & \\
\hline & & & & ISGR & $30(24)$ & $24(24)$ & $22(22)$ & $21(21)$ & S & 0.6 \\
\hline & SC & $33(26)$ & $30(24)$ & GR & $30(21)$ & $27(23)$ & $22(21)$ & $20(20)$ & $\mathrm{S}$ & $(0.6)$ \\
\hline & & & & SC & $31(26)$ & $26(23)$ & $23(22)$ & $22(26)$ & $M$ & \\
\hline \multirow[t]{4}{*}{ Nausea and vomiting $(\mathrm{nv})^{c}$} & IS & $6(13)$ & $7(15)$ & IS & $6(13)$ & $3(10)$ & $2(7)$ & $3(9)$ & & \\
\hline & & & & ISGR & $7(16)$ & $3(11)$ & $3(8)$ & $2(7)$ & S & 0.5 \\
\hline & SC & $6(12)$ & $6(13)$ & GR & $5(11)$ & $3(7)$ & $2(6)$ & $3(12)$ & & $(0.7)$ \\
\hline & & & & SC & $6(14)$ & $4(9)$ & $3(8)$ & $3(8)$ & & \\
\hline \multirow[t]{4}{*}{ Pain $(p a)^{b}$} & IS & $24(27)$ & $17(23)$ & IS & $18(25)$ & $17(23)$ & $17(20)$ & $15(20)$ & $\mathrm{S}$ & \\
\hline & & & & ISGR & $15(20)$ & $14(19)$ & $17(22)$ & $13(21)$ & M & 0.4 \\
\hline & SC & $23(26)$ & $18(21)$ & GR & $18(23)$ & $16(20)$ & $15(20)$ & $12(20)$ & M & $(0.8)$ \\
\hline & & & & SC & $19(19)$ & $17(22)$ & $18(23)$ & $15(23)$ & M & \\
\hline \multirow[t]{4}{*}{ Dyspnoea (dy) } & IS & $17(25)$ & $18(23)$ & IS & $19(24)$ & $23(26)$ & $18(25)$ & $19(27)$ & & \\
\hline & & & & ISGR & $18(21)$ & $20(25)$ & $16(23)$ & $16(23)$ & & 1.8 \\
\hline & SC & $17(23)$ & I8 (23) & GR & $18(21)$ & $15(19)$ & $19(20)$ & $15(24)$ & & $(0.1)$ \\
\hline & & & & SC & $19(24)$ & $21(23)$ & $16(23)$ & $17(26)$ & & \\
\hline \multirow[t]{4}{*}{ Insomnia (sl) ${ }^{\mathrm{b}}$} & IS & $27(3 \mid)$ & $20(26)$ & IS & $21(27)$ & $24(28)$ & $19(30)$ & $18(28)$ & S & \\
\hline & & & & ISGR & $19(24)$ & $19(28)$ & $18(24)$ & $18(25)$ & $\mathrm{S}$ & 0.5 \\
\hline & SC & $27(30)$ & $23(29)$ & GR & $22(27)$ & $16(24)$ & $20(24)$ & $16(23)$ & M & $(0.7)$ \\
\hline & & & & SC & $25(3 \mid)$ & $25(31)$ & $23(3 \mid)$ & $20(27)$ & $\mathrm{S}$ & \\
\hline \multirow[t]{4}{*}{ Appetite loss (ap) $)^{\mathrm{b}, \mathrm{c}}$} & IS & $18(30)$ & $9(20)$ & IS & $11(21)$ & $6(15)$ & $4(15)$ & $3(10)$ & $M$ & \\
\hline & & & & $I S G R^{d}$ & $8(19)$ & $5(14)$ & 7 (20) & $3(11)$ & $M$ & 0.3 \\
\hline & SC & $17(28)$ & $7(18)$ & $G^{d}$ & $5(14)$ & $3(9)$ & $6(14)$ & $5(14)$ & $M$ & $(0.8)$ \\
\hline & & & & SC & $10(21)$ & $6(17)$ & $5(16)$ & $4(13)$ & $M$ & \\
\hline \multirow[t]{4}{*}{ Constipation (co) } & IS & $9(23)$ & $8(19)$ & IS & $9(20)$ & $9(19)$ & $9(19)$ & $8(19)$ & & \\
\hline & & & & $I^{S G G}{ }^{f}$ & $7(19)$ & $8(21)$ & $9(20)$ & $9(20)$ & & 1.0 \\
\hline & SC & $10(23)$ & $9(21)$ & $G R^{f}$ & $5(14)$ & $9(19)$ & $9(18)$ & $6(17)$ & & $(0.4)$ \\
\hline & & & & SC & $12(26)$ & $12(23)$ & $10(24)$ & $10(24)$ & & \\
\hline Diarrhoea $(\mathrm{di})^{\mathrm{c}}$ & IS & $10(23)$ & $10(22)$ & IS & $11(24)$ & $6(14)$ & $5(16)$ & $4(12)$ & s & \\
\hline & & & & ISGR & $9(20)$ & $6(17)$ & $5(14)$ & 7 (20) & S & 0.7 \\
\hline & SC & II (22) & $10(20)$ & GR & $9(18)$ & $7(17)$ & $6(14)$ & $6(14)$ & S & $(0.6)$ \\
\hline & & & & SC & $11(22)$ & $8(19)$ & $6(16)$ & $8(17)$ & S & \\
\hline Financial difficulties $(\mathrm{fi})^{c, g}$ & IS & $5(16)$ & $8(2 \mid)$ & IS & $9(23)$ & $6(20)$ & $5(20)$ & $5(19)$ & & \\
\hline & & & & ISGR & $7(20)$ & $6(15)$ & $7(17)$ & $5(14)$ & & 1.0 \\
\hline & SC & $6(17)$ & $12(26)$ & GR & $9(21)$ & $7(17)$ & $5(13)$ & $5(19)$ & & $(0.4)$ \\
\hline & & & & SC & $14(30)$ & $13(26)$ & $11(26)$ & $7(19)$ & $S$ & \\
\hline
\end{tabular}

GR = group rehabilitation; IS = individual support; ISGR = IS and GR; SC = standard care. Statistically significant differences from baseline to 24 months (difference) are given as small (S), moderate $(M)$ or large $(\mathrm{L})$. ${ }^{\mathrm{F}} \mathrm{F}$ and $P$-value for IS-non-IS $\times \mathrm{GR}-$ non-GR $\times$ time interaction. d.f. $=3 / 954-963$. Improved from baseline to 3 months (ql, ef, and pa: $P<0.00$ I; sl: $P=0.012$; cf: $P=0.04$ ). Improved from 3 to 24 months (rf, sf, fa and nv: $P<0.00$ I; ql and di: $P=0.00 \mathrm{l}$; ef, ap and fi: $P=0.003 ;$ pf: $P=0.03$ ). ${ }^{\mathrm{d}}$ Time $\times \mathrm{GR}$ vs nonGR interaction for rf $(P=0.045)$ and ap $(P=0.007)$. ${ }^{~}$ Main effect of IS at 3-24 months $(P=0.046)$. ${ }^{f}$ Statistically significant differences between $G R$ patients and non-GR patients at baseline, $P<0.05$. ${ }^{8}$ Deterioration from baseline to 3 months $(P<0.00 \mathrm{I})$. 
Table 3 Mean values (s.d.) for HADS and IES for all groups (IS, $n=24 \mid$-9I; GR, $n=99-80 ;$ ISGR, $n=1 \mid$ 4-89; SC, $n=212-79)$ at five points of assessment

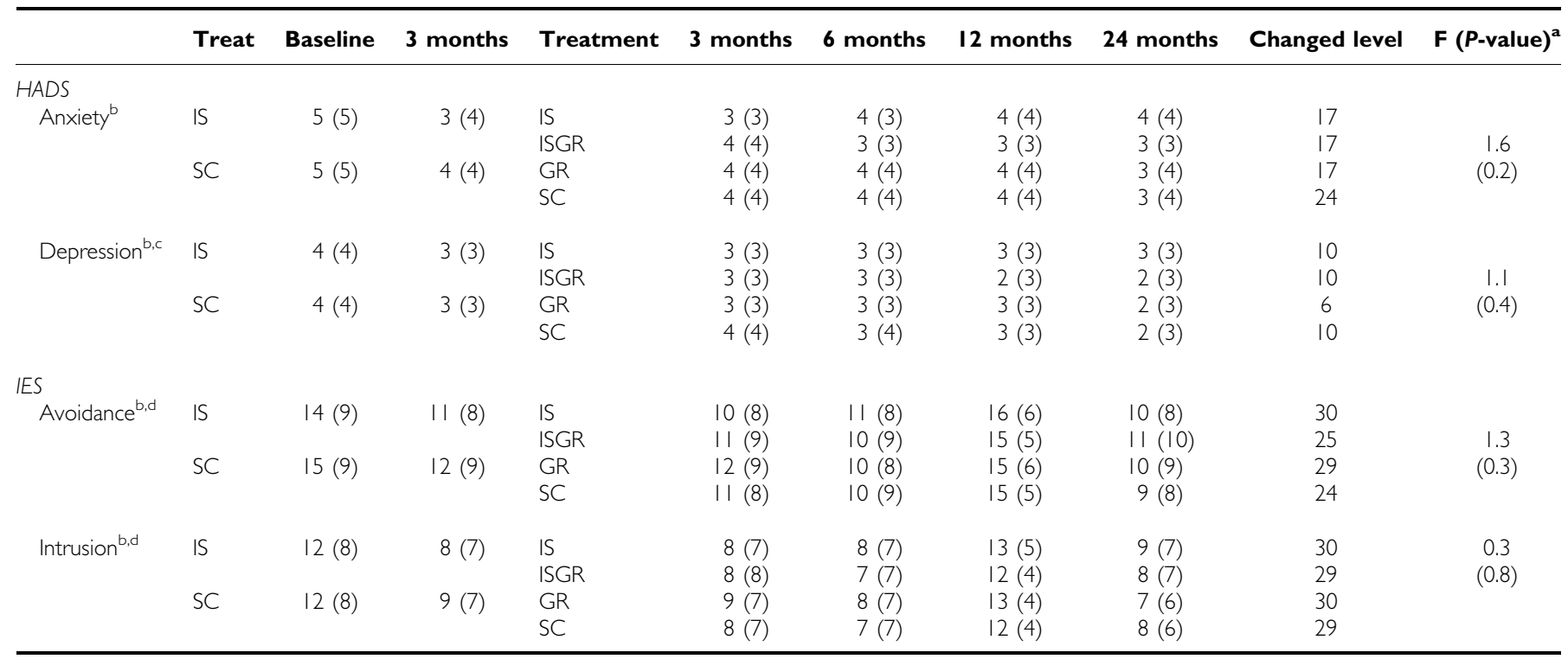

GR = group rehabilitation; HADS = Hospital Anxiety and Depression Scale; IES = Impact of Event Scale; IS = individual support; ISGR = IS and GR; SC = standard care. Changed level is the number of patients that changed level (improved) from baseline to 24 months according to recommended cutoff scores for the HADS and the IES a ${ }^{2}$ and $P$-value for IS-non IS $\times$ GR-non GR $\times$ time interaction. d.f. $=3 / 954-963$. ' $I$ mproved from baseline to 3 months, $P<0.00$ I. ${ }^{9}$ Improved from 3 to 6,12 , and 24 months, $P=0.014$. ${ }^{d} I \mathrm{mproved}$ from 3 to 6 months, worsened at 12 and improved again at 24 months, $P<0.001$.

Table 4 Mean values for EORTC QLQ C-30 subscales in the normal population and in the study sample at all points of assessments

\begin{tabular}{|c|c|c|c|c|c|c|c|c|c|}
\hline Subscales & Reference data & Baselir & $=457)$ & 3 mont & $=404)$ & 6 months $(n=386)$ & 12 months $(n=370)$ & 24 mon & $=339)$ \\
\hline Global health status & 75 & $64^{\mathrm{a}}$ & M & $70^{\mathrm{a}}$ & $\mathrm{s}$ & $72^{\mathrm{a}}$ & $73^{\mathrm{a}}$ & 75 & \\
\hline Physical functioning & 85 & $82^{\mathrm{a}}$ & & 83 & & 85 & 86 & 86 & \\
\hline Role functioning & 85 & $79^{\mathrm{a}}$ & & $81^{\mathrm{a}}$ & & 83 & 88 & $87^{b}$ & \\
\hline Cognitive functioning & 87 & $83^{\mathrm{a}}$ & & $85^{\mathrm{a}}$ & & $85^{a}$ & 86 & 86 & \\
\hline Social functioning & 90 & $83^{\mathrm{a}}$ & $\mathrm{S}$ & $86^{\mathrm{a}}$ & & 89 & $92^{\mathrm{b}}$ & 91 & \\
\hline Fatigue & 22 & $33^{c}$ & M & $30^{c}$ & $\mathrm{~s}$ & $26^{c}$ & 22 & 21 & \\
\hline Nausea and vomiting & 3 & $6^{c}$ & & $6^{c}$ & & 3 & 3 & 3 & \\
\hline Insomnia & 18 & $27^{c}$ & $\mathrm{~s}$ & $22^{c}$ & & $21^{c}$ & 20 & 18 & \\
\hline Appetite loss & 4 & $17^{c}$ & M & $8^{c}$ & $\mathrm{~s}$ & 5 & 5 & 4 & \\
\hline Constipation & 6 & $10^{c}$ & & $8^{c}$ & & $9^{c}$ & $9^{c}$ & 8 & \\
\hline Diarrhoea & 5 & $10^{c}$ & $\mathrm{~S}$ & $10^{c}$ & $\mathrm{~s}$ & $7^{c}$ & 6 & 6 & \\
\hline Financial difficulties & 7 & $5^{d}$ & & $10^{c}$ & & 8 & 7 & 6 & \\
\hline
\end{tabular}

Statistically significant differences given as small $(S)$, moderate $(M)$ or large (L) aHigher (better) mean value in the normal population, $P=0.02-<0.001$. ${ }^{b}$ Lower (worse) mean value in the normal population, $P=0.03-<0.001$. ' Lower (better) mean value in the normal population, $P=0.04-<0.001$. ${ }^{d}$ Higher (worse) mean value in the normal population, $P=<0.001$.

6 months, worsened at 12 months and improved again at 24 months. Pairwise comparisons revealed statistically significant differences between the 12-month assessment and the baseline, 3-, 6- and 24-month assessments.

\section{Comparison to HRQOL in the normal population}

The study participants reported lower HRQOL than the normal population at baseline (Table 4). However, the differences in mean values were small, and at 3 months, the study sample reported an HRQOL equal to that in the normal population. It should also be noted that the study participants reported less pain than the normal population at 3 , 6,12 and 24 months, and higher emotional functioning at 6,12 and 24 months.

\section{DISCUSSION}

The interventions evaluated in the SCR project did not improve newly diagnosed cancer patients' HRQOL or psychological wellbeing during a 24-month period after diagnosis, as compared with standard care. There were only a few statistically significant differences between treatment groups despite numerous analyses. The scores of several HRQOL domains rapidly improved with time, and at 3 months after diagnosis, the study sample reported an HRQOL comparable with the normal population.

The sparse interaction and main effects of treatments were small and inconclusive. However, the effects of time revealing an improvement in psychological well-being, pain and global quality of life (Tables 2 and 3 ) from baseline to 3 months in all treatment groups correspond well with results from earlier studies. These show, using the same measures that unscreened groups of cancer 
patients go through, a period of relatively rapid improvement with regard to HRQOL, including emotional functioning, after diagnosis and medical treatment (Nordin and Glimelius, 1997, 1998; Arving et al, 2007). Thus, the measures used in this study seem to be capable of detecting statistically significant and clinically relevant improvements with regard to these aspects.

Our results suggest that cancer patients generally manage to handle cancer-related concerns and side effects with the support available in standard care. However, several studies have shown that a substantial minority of cancer patients report high levels of distress and deteriorated HRQOL, which are not properly diagnosed and attended to (Burgess et al, 2005; Korfage et al, 2006). Therefore, there are reasons to explore the validity of our results. The following are noteworthy with regard to the external validity: (1) patients who rejected participation were significantly older than those who accepted participation, and (2) included patients who did not complete all assessments were older and more seriously diseased than those who completed the study. Patients with an advanced disease are likely to suffer more from emotional distress and deteriorated HRQOL and benefit from psychosocial interventions (Uitterhoeve et al, 2004). High age in cancer patients is associated with increased morbidity owing to concomitant diseases and impaired physical, social and cognitive functions (Extermann and Hurria, 2007). Hence, our results may suffer from a lack of data from subgroups of especially vulnerable patients, and the validity of our findings might thereby be limited to a group of relatively well-functioning patients. This assumption is supported by the presented data on HRQOL showing mainly improvements over time with only one exception for financial difficulties, which increased from baseline to 3 months, but improved again from 3 to 24 months.

When the SCR project was planned and initiated, the decision was not to screen patients, as this was not recommended at the time. On the contrary, great efforts were made to include as many patients as possible in the studied diagnostic groups, which we also succeeded in doing. However, the importance of screening to identify patients with a need for psychosocial support and to target interventions to such groups has been pointed out in several reports during the past decade (Sheard and Maguire, 1999; Sharpe et al, 2004). Thus, a recommendation for future projects aimed at evaluating the effects of psychosocial support should be to include screening and to target interventions only to those at risk for significant distress. On the other hand, there is no current consensus as to how to screen patients in need of an intervention for prolonged emotional distress (Mitchell, 2007).

The project was comprised of extensive interventions with the aim of maximising effects and increasing power. Individual support included individual psychological support, intensified primary health care and nutritional support for some patients, and implied extra contact with at least 2-3 different professionals, irrespective of the patients' need for support. Group rehabilitation comprised a total of nine sessions conducted by a psychologist,

\section{REFERENCES}

Aaronson NK, Ahmedzai S, Bergman B, Bullinger M, Cull A, Duez NJ, Filiberti A, Flechtner H, Fleishman SB, de Haes JC, Kaasa S, Klee M, Osoba D, Razavi D, Rofe PB, Schraub S, Sneeuw K, Sullivan M, Takeda F (1993) The European Organization for Research and Treatment of Cancer QLQ-30: a Quality-of-Life Instrument for Use in International Clinical Trails in Oncology. J Natl Cancer Inst 85: 365-375

Arndt V, Merx H, Stegmaier C, Ziegler H, Brenner H (2004) Quality of life in patients with colorectal cancer 1 year after diagnosis compared with the general population: a population-based study. J Clin Oncol 22: $4829-4836$

Arving C, Sjoden PO, Bergh J, Hellbom M, Johansson B, Glimelius B, Brandberg Y (2007) Individual psychosocial support for breast cancer physiotherapist and an oncology nurse. Both the individual psychological support and GR implied a visit by the patient to the SCR project agency, which for some patients was more than $100 \mathrm{~km}$ away from home. The data collection was also extensive. In addition to the measures included in the presented analysis, patients were asked to complete the Courtauld Emotional Control Scale (Watson and Greer, 1983) (baseline), the Miller Behavioural Style Scale (Miller, 1995) (baseline), the Reaction to the Diagnosis of Cancer Questionnaire (Frank-Stromborg, 1989) (baseline and 3 months), the Mental Adjustment to Cancer Scale (Watson et al, 1988) (all points of assessment), the Cancer Rehabilitation Evaluation System Short Form (Schag et al, 1991) (3, 6, 12 and 24 months) and another $2-5$ project-specific questionnaires during the 24-month follow-up period. In total, the data collection comprised 170-230 questions at each time of assessment. Thus, there is a risk that our efforts to maximise and measure the effects of the interventions meant that it was too strenuous for some groups of patients, with a need of support, to complete participation. This assumption is supported by the fact that older patients tended to reject participation and did not respond to questionnaires to the same extent as younger ones.

A possible Hawthorne effect should also be considered, as all study participants were contacted by phone at each point of assessment, to increase response rate and minimise missing data. The participants were contacted before the questionnaires were sent out, if they did not return them within in 2 weeks, and in cases of missing data on returned questionnaires. Hence, project participation may include numerous contacts with the project group, also for those randomised to standard care.

Comparisons to reference data may also contain some methodological difficulties (Hjermstad et al, 1998), that is the EORTC QOL-C30 has been developed for oncological patients and not for use in a normal population. However, it is widely recognised that the use of population-based reference values is relevant and provides an important aid in the interpretation of QOL scales (Michelson et al, 2000; Fayers et al, 2001).

In conclusion, many cancer patients manage to handle cancerrelated concerns and side effects with the support available in standard care. However, our findings seem to suffer from a lack of data from especially vulnerable patients and from a possible Hawthorne effect. Hence, it cannot be concluded that cancer patients have no need of additional psychosocial interventions. It is recommended that future projects include screening and targetfeasible interventions for those with the highest risks of significant psychological distress.

\section{ACKNOWLEDGEMENTS}

The study was supported by grants from the Swedish Cancer Society.

patients: a randomized study of nurse versus psychologist intervention and standard care. Cancer Nurs 30: E10-E19

Burge F, Lawson B, Johnston G (2003) Family physician continuity of care and emergency department use in end-of-life cancer care. Med Care 41: 992 - 1001

Burgess C, Cornelius V, Love S, Graham J, Richards M, Ramirez A (2005) Depression and anxiety in women with early breast cancer: five year observational cohort study. BMJ 330: 702

Extermann M, Hurria A (2007) Comprehensive geriatric assessment for older patients with cancer. J Clin Oncol 25: 1824-1831

Fayers P, Aronson N, Bjordal K, Groenvold M, Curran D (2001) Interpreting quality of life data: population-based reference data for the EORTC QLQ-C30. Eur J Cancer 37: 1331-1334 
Frank-Stromborg M (1989) Reaction to the Diagnosis of Cancer Questionnaire: development and psychometric evaluation. Nurs Res 38: 364-369

Hellbom M, Brandberg Y, Glimelius B, Sjödén PO (1998) Individual psychological support for cancer patients: utilisation and patient satisfaction. Patient Educ Couns 34: 247-256

Hjermstad MJ, Fayers PM, Bjordal K, Kaasa S (1998) Using reference data on quality of life - the importance of adjusting for age and gender, exemplified by the EORTC QLQ-C30 (+3). Eur J Cancer 34: 1381-1389

Horowitz M, Wilner N, Alvarez W (1979) Impact of Event Scale: a measure of subjective stress. Psychosom Med 41: 209-218

Johansson B, Berglund G, Glimelius B, Holmberg L, Sjödén P-O (1999) Intensified primary cancer care: a randomised study on home care nurse contacts. I Adv Nurs 30: 1137-1146

Johansson B, Holmberg L, Berglund G, Brandberg Y, Hellbom M, Persson C, Glimelius B, Sjödén P (2001) Reduced utilisation of specialist care among elderly cancer patients: a randomised study of a primary health care intervention. Eur J Cancer 37: 2161-2168

Kangas M, Henry JL, Bryant RA (2005) The course of psychological disorders in the 1st year after cancer diagnosis. J Consult Clin Psychol 73: $763-768$

Korfage IJ, Essink-Bot ML, Janssens AC, Schroder FH, de Koning HJ (2006) Anxiety and depression after prostate cancer diagnosis and treatment: 5-year follow-up. Br J Cancer 94: 1093 - 1098

May AM, Van Weert E, Korstjens I, Hoekstra-Weebers JE, Van Der Schans CP, Zonderland ML, Mesters I, Van Den Borne B, Ros WD (2008) Improved physical fitness of cancer survivors: a randomised controlled trial comparing physical training with physical and cognitivebehavioural training. Acta Oncol 47: 825-834

Michelson H, Bolund C, Nilsson B, Brandberg Y (2000) Health related quality of life measured by the EORTC QLQ C-30, reference values from a large sample of the Swedish population. Acta Oncol 39: 477-484

Miller SM (1995) Monitoring versus blunting styles of coping with cancer influence the information patients want and need about their disease. Implications for cancer screening and management. Cancer 76: 167-177

Mitchell AJ (2007) Pooled results from 38 analyses of the accuracy of distress thermometer and other ultra-short methods of detecting cancerrelated mood disorders. J Clin Oncol 25: 4670-4681

Newell SA, Sanson-Fisher RW, Savolainen NJ (2002) Systematic review of psychological therapies for cancer patients: overview and recommendations for future research. J Natl Cancer Inst 94: 558-584

Nordin K, Glimelius B (1997) Psychological reactions in newly diagnosed gastrointestinal cancer patients. Acta Oncol 36: $803-810$

Nordin K, Glimelius B (1998) Reactions to gastroentestinal cancer variation in mental adjustment and emotional well-being over time in patients with different prognoses. Psycho-oncology 7: 413-423
Osborn RL, Demoncada AC, Feuerstein M (2006) Psychosocial interventions for depression, anxiety, and quality of life in cancer survivors: meta-analyses. Int J Psychiatry Med 36: 13-34

Osoba D, Rodrigues G, Myles J, Zee B, Pater J (1998) Interpreting the significance of changes in health-related quality-of-life scores. J Clin Oncol 16: $139-144$

Persson CR, Johansson BBK, Sjoden PO, Glimelius BLG (2002) A randomized study of nutritional support in patients with colorectal and gastric cancer. Nutr Cancer 42: 48-58

Petersson L-M, Berglund G, Brodin O, Glimelius B, Sjödén P (2000) Group rehabilitation for cancer patients: satisfaction and perceived benefits. Patient Educ Couns 40: 219-229

Ravasco P, Monteiro-Grillo I, Vidal PM, Camilo ME (2005) Dietary counseling improves patient outcomes: a prospective, randomized, controlled trial in colorectal cancer patients undergoing radiotherapy. J Clin Oncol 23: $1431-1438$

Schag CA, Ganz PA, Heinrich RL (1991) Cancer Rehabilitation Evaluation System - short form (CARES-SF). A cancer specific rehabilitation and quality of life instrument. Cancer 68: $1406-1413$

Sharpe M, Strong V, Allen K, Rush R, Postma K, Tulloh A, Maguire P, House A, Ramirez A, Cull A (2004) Major depression in outpatients attending a regional cancer centre: screening and unmet treatment needs. Br J Cancer 90: 314-320

Sheard T, Maguire P (1999) The effect of psychological interventions on anxiety and depression in cancer patients: results of two-meta analyses. Br J Cancer 80: 1770 - 1780

Stanton AL, Revenson TA, Tennen H (2007) Health psychology: psychological adjustment to chronic disease. Annu Rev Psychol 58: $565-592$

Strong V, Waters R, Hibberd C, Rush R, Cargill A, Storey D, Walker J, Wall L, Fallon M, Sharpe M (2007) Emotional distress in cancer patients: the Edinburgh Cancer Centre symptom study. Br J Cancer 96: 868-874

Uitterhoeve RJ, Vernooy M, Litjens M, Potting K, Bensing J, De Mulder P, van Achterberg T (2004) Psychosocial interventions for patients with advanced cancer - a systematic review of the literature. $\mathrm{Br}$ J Cancer 91: $1050-1062$

van't Spijker A, Trijsburg RW, Duivenvoorden HJ (1997) Psychological sequelae of cancer diagnosis: a meta-analytical review of 58 studies after 1980. Psychosom Med 59: 280-293

Watson M, Greer S (1983) Development of a questionnaire measure of emotional control. J Psychosom Res 27: 299-305

Watson M, Greer S, Young J, Inayat Q, Burgess C, Robertson B (1988) Development of a questionnaire measure of adjustment to cancer: the MAC scale. Psychol Med 18: 203-209

Zigmond AS, Snaith RP (1983) The Hospital Anxiety and Depression Scale. Acta Psychiatr Scand 67: $361-370$ 